\title{
Supporting breast cancer survivors via text messages: reach, acceptability, and utility of EMPOWER-SMS
}

\author{
Anna C. Singleton ${ }^{1} \cdot$ Rebecca Raeside $^{1}$. Stephanie R. Partridge ${ }^{1,2}$. Justin Tat-Ko ${ }^{1}$. Stephanie Che Mun Sum ${ }^{1}$. \\ Karice K. Hyun ${ }^{1,3} \cdot$ Molly Hayes $^{1}$ - Clara K. Chow ${ }^{4,5,6,7}$. Aravinda Thiagalingam ${ }^{4,8} \cdot$ Katherine Maka $^{9,10}$. \\ Kerry A. Sherman ${ }^{8} \cdot$ Elisabeth Elder $^{9} \cdot$ Julie Redfern $^{1,6,5}$
}

Received: 4 July 2021 / Accepted: 26 August 2021 / Published online: 9 September 2021

(c) The Author(s), under exclusive licence to Springer Science+Business Media, LLC, part of Springer Nature 2021

\begin{abstract}
Purpose This study aims to evaluate the reach, usefulness, acceptability, and factors influencing engagement with a lifestylefocused text message intervention to support women's mental and physical health after breast cancer treatment.

Methods This study uses a mixed-methods process evaluation nested in the EMPOWER-SMS randomised controlled trial ( $n=160$; intervention $n=80$, wait-list control $n=80$ ). Data sources included screening logs, text message delivery software analytics, intervention feedback survey, and focus groups $(n=16)$, which were summarised thematically based on the framework approach.

Results A total of 387 women met the inclusion criteria ( mean $_{\text {age }} \pm \mathrm{SD}=59.3 \pm 11.6$ years). Participants who declined $(n=227)$ were significantly older than those who enrolled ( $n=160 ; 62.2 \pm 11.1$ vs $55.1 \pm 11.1$ years, respectively, $p<0.001)$. Most intervention participants $(64 / 80 ; 80 \%)$ completed the end-of-study survey, reporting the messages were easy to understand $(64 / 64 ; 100 \%)$, useful $(58 / 64 ; 91 \%)$, and motivating $(43 / 64 ; 67 \%)$. The focus groups $(n=16)$ revealed five factors influencing engagement: (i) feelings of support/continued care, (ii) convenience/flexibility of message delivery, (iii) weblinks, (iv) information from a credible source, and (v) options to save or share messages.

Conclusion A lifestyle-focused text message program was acceptable and useful for women after breast cancer treatment. However, text messaging may be a barrier for women aged over 68 years. Suggestions for program improvements included delivering the program to patients with other cancers, during all stages of treatment, and including more weblinks in text messages.

Implications for Cancer Survivors

Text message programs offer a low-cost way to deliver post-treatment health support to breast cancer survivors in a noninvasive way. Text messages can improve patient-health professional communication and were found to be acceptable and useful.
\end{abstract}

Keywords Breast cancer survivor $\cdot$ Mobile health $\cdot$ mHealth $\cdot$ Supportive care $\cdot$ Text messaging $\cdot$ Process evaluation

Anna C. Singleton

anna.singleton@sydney.edu.au

1 Engagement and Co-design Research Hub, School of Health Sciences, Faculty of Medicine and Health, University of Sydney, Level 6 Block K Westmead Hospital, Westmead, Sydney, Australia

2 Prevention Research Collaboration, Charles Perkins Centre, University of Sydney, Sydney, Australia

3 Department of Cardiology, Concord Repatriation General Hospital, Sydney, Australia

4 Westmead Applied Research Centre, Faculty of Medicine and Health, University of Sydney, Sydney, Australia
5 Department of Cardiology, Westmead Hospital, Sydney, Australia

6 George Institute for Global Health, University of New South Wales, Sydney, Australia

7 Research Education Network, Western Sydney Local Health District, Sydney, Australia

8 Centre for Emotional Health, Department of Psychology, Macquarie University, Sydney, Australia

9 Department of Physiotherapy, Westmead Hospital, PO Box 533, Sydney, Australia

10 Westmead Breast Cancer Institute, Westmead Hospital, PO Box 143, Westmead, Sydney, Australia 


\section{Introduction}

Breast cancer is the most common cancer among women globally [1] with $80-90 \%$ of women living at least 5 years after treatment. However, breast cancer treatment can negatively impact women's mental and physical health for years after treatment has finished [2,3]. Structured post-treatment support is sparse [4] and available services are inaccessible to many patients due to in-person delivery, limiting attendance for those in rural and remote areas, and/or those with work or family responsibilities, financial stressors, or mobility issues [5]. Moreover, the recent global novel coronavirus disease (COVID-19) pandemic has caused widespread and unpredictable cancellations of in-person cancer-related appointments [6, 7]. There is an urgent need for accessible, inexpensive, and easily scalable support strategies.

Text message interventions are an emerging strategy to provide health support remotely. Research has found such programs effective for improving a range of mental and physical health outcomes, including depression [8], blood pressure, low-density lipoprotein cholesterol, and exercise levels $[9,10]$ in patients with chronic diseases, and they may be useful for providing support for stress and anxiety during COVID-19[11]. Moreover, patients find text messages convenient, flexible, and a non-intrusive way to receive support [12]. Text messages are inexpensive to deliver compared to in-person support and can help patients feel more connected to their healthcare team $[12,13]$. However, a systematic review found that there were no randomised controlled trials (RCTs) of text message interventions aimed at improving mental or physical health outcomes for patients during or after breast cancer treatment [14]. Pre-post studies and one recent RCT found that text message interventions may help patients with breast cancer adhere to endocrine therapy tablets $[19,20]$ and maintain weight loss [21]. However, evidence for a text message intervention's acceptability and usefulness among patients with breast cancer is needed.

Co-design of health services has been found to improve end-user satisfaction and engagement $[15,16]$. However, a recent review found that electronic health (eHealth) interventions to support women's health during and after breast cancer treatment are rarely co-designed with end-users, though it is highly encouraged [14, 17-19]. For text message interventions, qualitative assessments of user uptake and service preferences (e.g. delivery timing, frequency, content) are critical for service optimisation [12]. Our team recently co-designed a text message intervention (EMPOWER-SMS) with breast cancer survivors, researchers, and health professionals to support women's mental and physical health after breast cancer treatment [20], which was subsequently tested in a 6-month RCT compared to usual care [21]. The aim of the present study was to investigate the potential reach, perceived usefulness and acceptability, and factors influencing engagement with the EMPOWER-SMS intervention. Moreover, the study aimed to elucidate barriers and enablers to implementation and women's recommendations for intervention improvements.

\section{Methods}

\section{Study design}

A mixed-methods process evaluation nested within a 6-month text message intervention was conducted in the EMPOWER-SMS randomised controlled trial $(N=160$; intervention $n=80$; control $n=80$ )[21]. EMPOWER-SMSRCT aimed to improve self-efficacy, quality of life (QOL), and mental (depression, anxiety, stress) and physical (body mass index [BMI], physical activity, healthy eating, endocrine therapy medication adherence) health outcomes after breast cancer treatment compared to usual care.

\section{Participants and recruitment}

Participants were recruited from a large breast cancer institute in Sydney, Australia, with a diverse cultural population. Participants were eligible to participate if they were women (age $>18$ years) who completed active breast cancer treatment (surgery and/or chemotherapy and/ or radiotherapy - could still be taking endocrine therapy medication) within 18 months, had sufficient English skills to provide informed consent, and owned a mobile phone. All participants were required to attend two, one-hour inperson (baseline, 6-month follow-up) study visits to complete surveys and body composition measurements with a researcher blinded to the participant's group allocation. Due to the COVID-19 restrictions, 4/160 (2.5\%) participants were recruited over the phone and 79/160 (49\%) completed follow-up visits over the phone. A subset of intervention participants were invited via telephone to attend a focus group to provide a more in-depth understanding of the perceived barriers and facilitators to engaging with and receiving the text message intervention. Participants were randomly selected from intervention participants using an online number generator and then purposefully selected for ethnically, culturally, and socioeconomically diverse backgrounds to ensure a variety of views were explored.

\section{Intervention}

The text message intervention and its development process are described elsewhere [20,21] Briefly, participants 
received four semi-personalised text messages per week at random times (either $9 \mathrm{am}, 12 \mathrm{pm}, 3 \mathrm{pm}$, or $6 \mathrm{pm}$ ) on random days (Monday to Saturday). Message content included encouragement, support, and practical tips regarding mental health, physical activity, healthy eating, medication adherence and side effects, social and emotional support, and general breast cancer information. Some messages included links to websites with further information, which were selected during the intervention co-design process (breast cancer survivors, health professionals, researchers) and included Australian not-for-profit organisation (e.g. Breast Cancer Network Australia, National Heart Foundation of Australia) or government websites (e.g. Cancer Australia). Example text messages have been previously published [20]. The program was one-way (replies discouraged). However, for safety, a health counsellor monitored replies and kept a log of reply content. Participants could opt out any time by replying "stop". All text messages were delivered free of charge.

\section{Data sources for mixed-methods analysis}

The data sources for the mixed-methods analysis included:

1. Screening logs: to understand the reach and potential generalisability of a text message intervention, a screening $\log$ was kept of reasons for declining participation (March 2019-2020) and participants' age (years). The log was stored in a secured research database.

2. Text message delivery software analytics: text messages were delivered using automated software (April 2019 to November 2020). To assess if the intervention was delivered as planned, the number of text messages that were sent, successfully delivered, not delivered successfully ("bounced"), or resulted in an "opt out" were recorded.

3. End-of-study intervention feedback survey (intervention participants only): during a 6-month follow-up visit with a blinded researcher, intervention group participants completed an end-of-study intervention feedback survey, which included four free-text questions regarding participants' most and least liked messages, suggestions for program improvements, and general comments.

4. Focus groups $(n=16)$ : explored barriers and facilitators to engaging with and receiving a text message intervention; insights regarding key topics such as perceived utility, acceptability, and health impacts of the intervention; and preferred program delivery length, timing, frequency, and content, factors influencing engagement with the program and suggestions for program improvement. The focus groups followed standard methods, including an experienced facilitator (JR), scribes (AS, $\mathrm{RR}$ ), the setting of ground rules, and audio recordings (two devices)[22]. However, due to the COVID-19 pandemic and the prohibition of in-person gatherings [23], the focus groups were conducted via teleconference. The facilitator had over 12-year experience in chronic disease management research but was not known to the research participants. Additional focus groups were conducted until no new themes emerged based on ongoing analysis (i.e. thematic saturation). Participants were reimbursed $\$ 20$ for their time.

\section{Analyses}

Continuous data (e.g. age) were summarised as means and standard deviations, and categorical data were summarised by frequencies and percentages. The mean age (years) of women who enrolled or declined study participation and the mean time (days) between completing active treatment and enrolling or declining were compared using independent samples $t$ test. Free-text responses were independently parallel coded into themes by two researchers (AS, JTK). Focus groups were digitally recorded and transcribed verbatim and were independently parallel coded into themes in two iterative stages by two researchers (AS, SCMS) based on the framework approach [24] using NVivo version 12.0 (QSR International). The coders were experienced researchers with expertise in psychology, chronic disease management, and mixed-methods research approaches. Codes were constantly compared with previously coded data and combined or expanded until no new concepts or themes emerged. Resulting codes were then evaluated by a third independent researcher (JR). Disagreements were discussed until an agreement was reached with quotations used to illustrate opinions.

\section{Results}

\section{Potential reach and generalisability of text message intervention}

A total of 387 women met the study inclusion criteria and had a mean ( \pm standard deviation) age of $59.3 \pm 11.6$ years (Fig. 1). A total of 160 women enrolled in the study and 227 declined, mainly due to "personal reasons" (157/227; $70 \%$; see Fig. 1). Participants who declined were significantly older than those who enrolled $(62.2 \pm 11.1 \mathrm{vs}$ $55.1 \pm 11.1$ years, respectively, $p<0.001$ ). The text message technology was a barrier for some older women because they "did not own a mobile phone" $(17 / 227 ; 7 \%$; aged $77.71 \pm 8.21$ years, $p<0.001)$, "did not know how to open/ read text messages" $(9 / 227 ; 4 \%$; aged $76.78 \pm 8.03$ years, $p<0.001)$, or "rarely turned phone on" $(7 / 227 ; 3 \%$; aged $68.14 \pm 7.27$ years, $p<0.05)$. Participants who declined 
Fig. 1 Participation flow diagram and reasons for nonparticipation

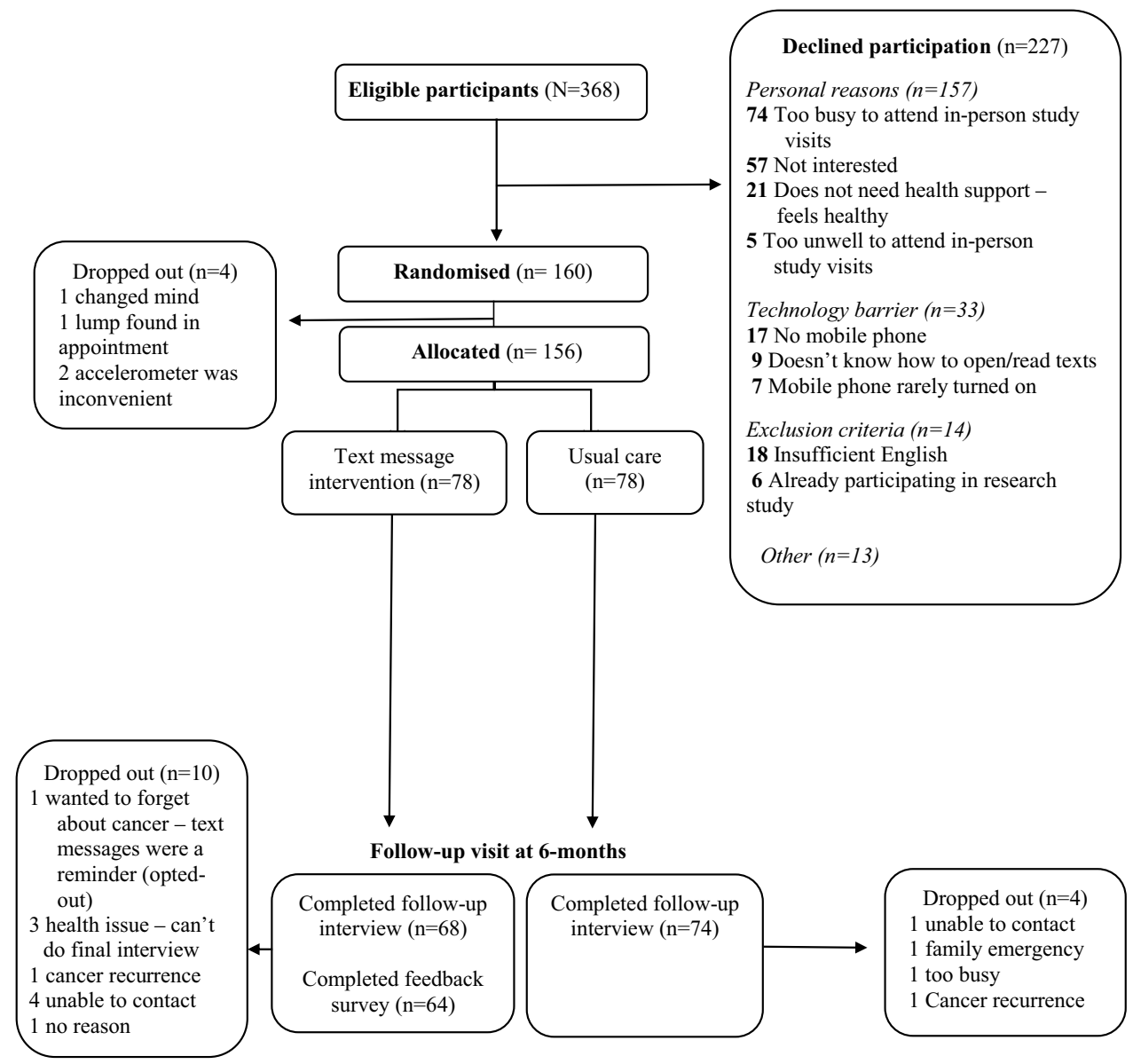

also finished active treatment more recently than those who enrolled $(228.76 \pm 156.30$ days vs $245.43 \pm 152.24$ days, respectively, $p<0.01)$.

\section{Evaluation of program delivery, usefulness, and acceptability}

Eighty participants were randomised to receive the text message intervention. However, 2/80 (2.5\%) dropped out prior to beginning the intervention (Fig. 1). A total of 8061 text messages were sent (April 2019 to November 2020) to intervention participants; 7925/8061 (98.3\%) were delivered successfully and 136/8061 (1.7\%) bounced. Two participants opted out after 2 months and 6 days, respectively, stating they "did not want to be reminded of cancer".

Of the 80 participants randomised to the intervention group, 64/80 (80.0\%) completed the end-of-study intervention feedback survey (Fig. 1) and 16 participated in the focus groups. Participant demographic data are presented in Table 1. The focus groups and end-of-study feedback survey free-text responses further elucidated participants' perceptions of the usefulness and acceptability of the program's (i) message content, (ii) message delivery frequency, (iii) message timing, (iv) program duration, and (v) one-way communication:

Message content The feedback survey free-text responses and focus groups revealed that the message content was easy to understand, useful, and acceptable. Participants preferred varying, positively toned message content regarding achievable lifestyle changes, practical advice, and self-care (see Box 1).

Box 1 Quotes illustrating participants' perceptions of message content and usefulness

Theme 1. The message language is accessible to everyone

"All those messages can apply to everybody" (Female, age 60, ID11)

"I found the information received was very informative and easy to understand" (Female, age 64, ID99)

"I, most of the time, followed the links that you provided. Read the information which I think is very clear, very straightforward, very understandable by the general population" (Female, age 66, ID13)

Theme 2. The lifestyle change (diet, exercise, mental health, medication adherence) was useful 
Table 1 Feedback survey $(n=64)$ and focus group $(n=16)$ participants' demographic and medical history

\begin{tabular}{|c|c|c|}
\hline & Feedback survey $(n=64)$ & Focus groups $(n=16)$ \\
\hline Age, mean (SD); range & $55.20(11.15) ; 29-76$ & $55.32(11.31), 36-67$ \\
\hline \multicolumn{3}{|l|}{ Ethnicity, $n(\%)$} \\
\hline White & $30(46.9)$ & $8(50.0)$ \\
\hline Asian (including south/southeast Asia) & $21(32.8)$ & $7(43.8)$ \\
\hline Other & $13(20.3)$ & $1(6.2)$ \\
\hline \multicolumn{3}{|l|}{ Region of birth, $n(\%)$} \\
\hline Australia/New Zealand & $33(51.6)$ & $9(56.2)$ \\
\hline Asia (including south/southeast Asia) & $20(31.2)$ & $7(43.8)$ \\
\hline Other & $11(17.2)$ & $0(0)$ \\
\hline \multicolumn{3}{|l|}{ Highest level of education, $n(\%)$} \\
\hline$\leq$ year (grade) 10 & $11(17.2)$ & $0(0)$ \\
\hline High school (year/grade 12) certificate & $7(10.9)$ & $0(0)$ \\
\hline Diploma/technical degree & $18(28.1)$ & $6(37.5)$ \\
\hline University or post-graduate degree & $27(42.2)$ & $10(62.5)$ \\
\hline \multicolumn{3}{|l|}{ Employment status, $n(\%)$} \\
\hline Working (part-time or full-time) & $45(70.3)$ & $12(75.0)$ \\
\hline Retired, unemployed, or homemaker & $19(29.7)$ & $4(25.0)$ \\
\hline Married/De Facto, $n(\%)$ & $44(68.8)$ & $11(68.8)$ \\
\hline Has (a) Child(ren), $n(\%)$ & $54(84.4)$ & $13(81.2)$ \\
\hline Child(ren) aged $>19$ years & $35(64.5)$ & $6(46.2)$ \\
\hline Child(ren) aged $\leq 18$ years & $19(35.2)$ & $7(53.8)$ \\
\hline \multicolumn{3}{|l|}{ Breast cancer treatment, $n(\%)$} \\
\hline Surgery & $64(100)$ & $16(100)$ \\
\hline Chemotherapy & $40(62.5)$ & $8(50.0)$ \\
\hline Radiotherapy & $59(92.2)$ & $15(93.8)$ \\
\hline Endocrine therapy tablets & $40(62.5)$ & $12(75.0)$ \\
\hline $\begin{array}{l}\text { Time between completing active breast cancer } \\
\text { treatment and enrolment (months), mean (SD) }\end{array}$ & $8(5)$ & $6(5.45)$ \\
\hline
\end{tabular}

"My initial reaction is that I didn't do anything different because of these messages. But actually when I think about it, now I am nearly vegetarian, I don't drink [alcohol] very much, I'm very conscious of not eating too many cured meats. So even though maybe it didn't have an immediate impact, it had a long-term impact on the way that I think about the things that I can do to make sure it [cancer] doesn't come back" (Female, age 37, ID40)

"It motivated me to walk a bit more. I started walking, just $10 \mathrm{~min}$, 20 min. Doing little exercises. Now I am making time for what I eat. How was like nutrition, food and going for a walk. At least those simple things I have started doing" (Female, age 57, ID71)

"I found like, I've had a bad day for whatever reason, and the message came through and they're just like 'remember that exercise is important, but every little bit counts' so you know, 'did you climb those stairs or did you just go around the block?' and I'm just like, well, yep, I manage that! So, I wasn't completely negative in my own self, so then [I'd] have that positive comeback" (Female, age 47, ID152)

"Every message sent was useful as I was informed of new ways of dealing with things like moods due to hormone medication and all other new things that you have to deal with after breast cancer" (Female, age 41, ID89)

Theme 3. The importance of message variety and practical advice

\footnotetext{
"There was a fair bit of time, maybe several weeks, between similar [topics] So there's a certain cycle in it and a certain reinforcement and slightly different slants from the same topics, it maybe took you to a different website etc.. So I think that was quite good really" (Female, age 66, ID13)

"I thought there was a good mix of tips \& ideas" (Female, age 67, ID114)

"I think it was a good package, as well as the links you know how to improve our general health, about self-care and about those simple messages, and also there's quite a few messages regarding reminding you it's important to take your anti-hormone tablet, why do you need to do and there's a couple of messages on how to deal with hot flushes as part of the medication, side effects of medication and all that. So it's just a whole package of everything reminding you of everything that all this can help with your symptoms" (Female, age 60, ID11)

"being on tamoxifen sometimes [...] you just don't wanna get up, don't want to exercise, all I do was sleeping and I've got at that time the [message] about exercising. I was finding exercising [a] bit of a chore and you sent out one [message] saying to 'do it in intervals'... 'do it $10 \mathrm{~min}$ in the morning, $10 \mathrm{~min} . .$. " and I started doing that and found that really good. So it was empowering me to actually do stuff" (Female, age 41, ID89)
}

Theme 4. The impact of positively toned and self-care messages 


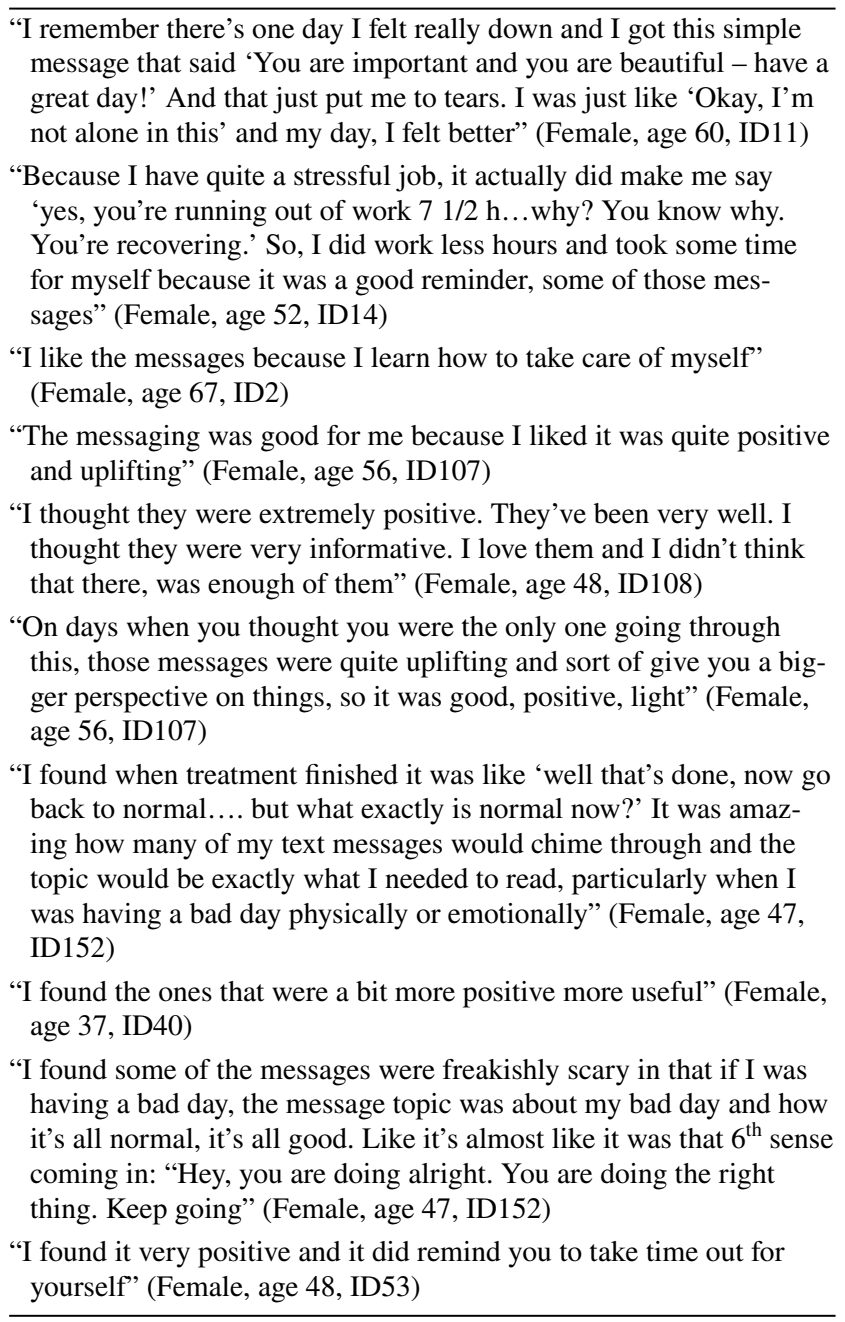

Message frequency Most participants felt that the number of messages they received per week was acceptable. However, it was personal preference:

I don't really know whether it's too many or not enough, when you're feeling down and you really need that extra support, it's never going to be enough" (Female, age 60, ID11)

Although participants received only four messages per week, most participants in the focus group felt that they were receiving messages "every day":

It was nice to get one everyday pretty much (Female, age 51, ID132)

Message timing The consensus among focus group participants was that the message timing was acceptable, and they read the messages when it was convenient for them:

The timing didn't bother me. I would prefer it not first thing in the morning, but 9, 10 o'clock, then [it's] something to think about and reflect through the day, whereas if you get it in the evening, you only got a short time to be awake and go to sleep and forget about it..." (Female, age 66, ID13)

I did get quite a few around the six o'clock mark, but I actually got a few at 9:00 AM and at 12:00PM as well. So, usually it wasn't until the evening that I was reading them" (Female, age 47, ID152)

Program duration The consensus among the end-of-study feedback survey and focus group participants was that they would like the option to continue the program. However, many suggested they would like to choose how long they continue and the number of messages per week:

I would have liked it to continue. I miss the messages (Female, age 58, ID47)

To extend it to 12 months or longer if possible would be awesome (Female, age 53, ID140)

A very worthwhile program! After the initial run there could be some follow up messages a year later, a reminder to keep focused on our health (Female, age 51, ID158)

One-way communication Although most participants found the one-way communication acceptable, the focus groups elucidated that participants would like the option to reply to seek advice from a health professional if needed. For example:

The ability to reply to that text with a question... if [the message] came from a breast care nurse or somebody that could help (Female, age 51, ID132)

If you can add on live chat, [that] will be great. There are times we want to get someone to help sort out a bit of confusion and no one is there to talk to (Female, age 64, ID97)

\section{Factors influencing engagement}

Overall, the program was well-liked by participants and provided a sense of support:

The fact that someone is keeping in touch is useful because after the last day of treatment it's easy to think 'Well, is that it then? What do I do now?' The program can make people feel remembered. A follow up besides a doctors visit is friendly \& useful (Female, age 67, ID114)

This program helped improve my life. I am so thankful I did it (Female, age 58, ID53)

The feedback survey free-text responses and focus groups revealed five factors influencing engagement with the program: (i) feelings of continued care and support, 
(ii) convenience and flexibility of messages delivery, (iii) weblinks with additional information and resources, (iv) receiving information from a credible source, and $(\mathrm{v})$ the option to save or share messages (see Box 2).

Box 2 Quotes illustrating factors influencing participants' engagement with the text message program and suggestions for improvement

\section{Theme 1. Feelings of continued care and support}

"I used to see [the messages] and it's like another friend for me and I never get annoyed or anything. Just like my friend is sending me messages" (Female, age 57, ID71)

"Very positive. I felt like a friend was keeping an eye on me and some messages arrived at the perfect time when I was feeling a bit low, they helped" (Female, age 43, ID124)

"I found the messages very positive and almost like a silent supportive partner" (Female, age 49, ID90)

"[I liked] messages that reminded me that I wasn't alone, that how I was feeling was a normal part of the process" (Female, age 55, ID145)

“It just made you feel as though you weren't completely alone because everyone has different circumstances. Personally, I don't have a partner, I live alone so I was pretty much here by myself most of the time" (Female, age 51, ID132)

"I think it continued the care from Westmead [Breast Cancer Institute]. It was just another liaison to it. I felt like the care from the Breast Cancer Institute did not stop. It just continued with these messages..." (Female, age 41, ID80)

"There's quite a few messages that remind you that you can always contact the breast care nurse to talk to and for any additional help and support. And that's like constantly reminding you that you're not alone, that there's always someone that you can call. It encouraged you to approach them more often, especially with the messages sometimes it might jog your memory like 'ah', to ask this of my GP or the BCI" (Female, age 60, ID11)

"I think this programme is really good and really support[ive]. I've done my radiotherapy, it's already 2 years. I've been just staying at home, just get off at the workplace, so not many people to see everyday. Sometimes I just feel that the life is really, really boring and I need some information. I need something to contact with the outside and everyday when I read [the message] it just makes me a little happy" (Female, age 50, ID61)

\section{Theme 2. Convenience and flexibility of message delivery}

"[The message] usually [came] around dinner time, but you can choose when to sit down and read them" (Female, age 58, ID53)

"It's our choice whether to read those messages or wait 'til a better time to read it. There's no harm to read it later in the day or the next day" (Female, age 60, ID11)

"Yeah, I've kept them all. I was just scrolling through I think the first, probably 10 or so. I truly wanted to read it straight away depend on where I was. But then once I started to see that it was the research study, I knew that it was an important one to read, but I could read it in my time later" (Female, age 47, ID152)

Theme 3. Links to websites with additional information

"I really liked getting the links because it took you straight there [to the information], it's not something that you had to put effort into research, some days you just need to be spoon fed" (Female, age 51, ID132)

"I thought the resources that they linked you to are really helpful" (Female, age 48, ID100)
"The big benefit from it [was the link to the] exercise program for post treatment breast cancer lady. I got that message [with] the information from your message and I contact them and I joined their program. It's really, really helpful and give me some social life and meet some other women, [with a] similar experience as mine" (Female, age 50, ID61)

"When you become a patient yourself, you need that additional support and one of the things that on the link was the Cancer Council support group and I thought okay... I was really iffy about it but then I joined and I actually found it very useful and very supportive" (Female, age 60, ID11)

\section{Theme 4. Receiving information from a credible source}

"Usually if I Google stuff it would come as American information and I just wasn't sure if I was reading the right thing but because the link came from you, I thought "Oh, okay, that's the right thing..." (Female, age 41, ID80)

"You are Googling around and you're thinking 'oh is this a good source of information? Or some random person who doesn't know what they're talking about?' So that was really handy to get pushed towards places that I presumably can trust, cause you're from [the] Uni" (Female, age 72, ID74)

Theme 5. Option to save or share the messages

"I've kept them all, saved them so I can go back if I want to" (Female, age 47, ID152)

"I don't mind if it's long or short because what I do is save it to my phone and whenever I want to read it, I'll just go back to my files" (Female, age 67, ID2)

"I can save them so I can go back if I want to. And I know that some ladies I've met through treatment who aren't part of this study... we'll have a conversation, I'm just like, 'oh, have you thought of?' Whatever the link was that I was told about. So, they've gone, 'oh yeah, I had a look at that ages ago. I'll have a look at it again now", (Female, age 47, ID152)

"We did receive a message regarding medication and regarding side effects and things like that. So these messages made me think that it wasn't just me just making up these pains and aches and things like that. So I did everything you said. I asked my GP. I went to the oncologist'" (Female, age 52, ID14)

"It was a special thing for myself" (Female, age 41, ID89)

Theme 6. Suggestions for improvement

\section{Program available to all cancer patients}

"The program was helpful and extremely positive. In my opinion I think this program should be extended to all cancer patients not just breast cancer" (Female, age 48, ID108)

\section{Program available during and after cancer treatment}

"Program would be great from the beginning of treatment. I started with chemo and receiving the relevant text messages about chemo at the time would have been helpful" (Female, age 47, ID152)

"I wish this was more available when first diagnosed. I was so depressed and unable to sleep. These SMS are very helpful" (Female, age 67, ID57; text message reply)

"[After treatment, messages] about not getting cancer again or how to keep yourself from it. Those [were the] information I was more interested to look at" (Female, age 46, ID77)

More weblinks, including at-home exercises or online programs (COVID19-safe)

"I love the links. I would like that links every message, cause it's entirely up to you whether you want to click on that link or not" (Female, age 52, ID14) 
"More links to services, videos of gentle exercise classes that can be done at home" (Female, Age 52, ID132)

Promote the program via clinicians and social media

"The breast care nurse. I think it's probably the person who hopefully has more time in the clinic. I know the clinic is frantically busy to sit down and talk to people and I think that's more appropriate than the doctor. I didn't have much to do with my GP [general practitioner] during that actual treatment" (Female, age 66, ID13)

"BCI [ Breast Cancer Institute] would be the best because they know you as a patient, one on one" (Female age 56, ID107)

"From a GP or medical centre, [they] have a lot of practice nurses. The more staff that knows about this program, then they can reach out to a lot more people" (Female, age 60, ID11)

"[Social media] is probably a good place to reach people because Facebook tends to be populated now by people more around our age. I'd like to know the success rate [of recruitment in-person] compared to Facebook because a lot of people are very sceptical about clicking on things on Facebook too. And I think in waiting rooms and in the BCNA [Breast Cancer Network Australia] journal you get" (Female, age 51, 132)

\section{Suggestions for improvement}

Participants felt that the program should be available to all patients with cancer, tailored to each type of treatment (surgery, chemotherapy, radiotherapy, post-active-treatment) and more weblinks, including COVID-19-safe suggestions for at-home or online service options (see Box 1). Participants also wanted to hear about the program from someone who they feel is credible(e.g. breast care nurse, oncologist, breast cancer institute, GP).

\section{Paying for the program}

Participants wanted the program as part of standard care and would be willing to pay. However, due to ongoing financial stresses associated with breast cancer, the program ought to be subsidised by the government, medical insurance, telecommunication companies, or not-for-profit organisations:

We haven't had to pay for [treatment-related] things. But you often would have to give up work, and it doesn't mean that you've been able to have paid leave for time that you had [off]. So, if you've got health insurance and they will cover it, could you please use that? If you don't, well, then it is a support service that the government should [pay for]. 'Cause within reason, keeping us physically and mentally sane is cheaper than us going physically and mentally insane (Female, age 47, ID152)

I think you will have better uptake if the individual doesn't have to pay for it. Maybe it could come from the cancer society or the Breast Cancer Institute, or breast cancer charities (Female, age 66, ID13)
It would be good to get a sponsor that could help, say [telecommunication companies] (Female, age 51, ID132)

\section{Discussion}

This mixed-methods process evaluation found that a text message intervention was wide-reaching, delivered positively toned motivational health support in a nonintrusive way, and helped participants feel cared for. The text messages were delivered as planned, with minimal intervention-related barriers to participation and low attrition rates. Participants provided positive feedback about the program, finding it acceptable, useful, and motivating. Importantly, participants felt strongly about messages that were positively toned and supported self-care and mental health. These message instilled feelings of support and continued care from health providers, despite the one-way (no replies) nature of the program. Several factors influencing engagement were identified including the convenience and flexibility of text message delivery and optional weblinks, the perception of credible sources of information, and the option to save and share messages. Some participants used the program information to prompt further discussions with their care team, whereas the majority kept the program to themselves as a "personal experience". Suggestions for program improvement and enablers for implementation included adding more optional weblinks (including COVID-19-safe resources), delivering the program from credible medical staff (e.g. GPs, oncologists, breast care nurses), allowing optional two-way communication, making the program available to all cancer patients during and after treatment, and subsidising the program to reduce financial barriers.

The text message intervention may be a wide-reaching and acceptable way to deliver support and continued care to breast cancer patients between medical visits. The text message intervention was only a barrier for some (33/387; $8.5 \%$ ) older patients. As mobile phones become ubiquitous among people aged over 65 years [25], this barrier should be minimised. Patients who enrolled in the study expressed that they enjoyed receiving text messages because they felt supported and connected to the breast cancer institute. Moreover, participants wanted to receive the messages during and after treatment with the option to reply with questions, to facilitate provider-consumer communication, reduce stress between medical visits, and promote a seamless transition to post-treatment care. Two-way communication would also allow tracking of self-set goals, which were encouraged within the EMPOWER-SMS program [20]. Currently, post-treatment health support programs are not consistently 
available or accessible to patients.[4] However, a recent meta-analysis found that electronic health (eHealth) support strategies, namely, interactive websites, for patients during and after breast cancer treatment improved quality of life, distress, and fatigue [14]. Interventions with an option to electronically (email, text message, instant message) contact a health professional increased user engagement and acceptability. Supportive telephone calls for cancer survivors between medical visits have also been found to be convenient, accessible in terms of space and time, and helpful for avoiding "slipping through the net" of the healthcare system [26]. The current study's text message intervention showed similar results. However, text messages are less cost- and resource-intensive $[13,27]$, which may facilitate accessibility and scalability of continued patient care between medical visits.

Patient-driven personalisation through optional intervention features may be key to user acceptability and engagement. Participants in the current study offered important suggestions for intervention improvement, such as wanting a choice in how long the program lasted, the number of messages per week, and the time-of-day messages were received. Moreover, participants preferred program features that were optional, such as clicking on a weblink or saving and sharing messages. Although there is mixed evidence that personalisation of text message interventions improves effectiveness $[28,29]$, there may be a distinction between researcher- and user-driven personalisation. From a psychological perspective, making a choice can fundamentally improve a person's preference for whatever was chosen [30]. Making an informed choice can also improve a person's sense of control or self-efficacy [31,32], which has been found to improve health outcomes [33]. In a study aimed at reducing cardiovascular (CVD) risk factors (physical inactivity, medication non-adherence, unhealthy diet), providing participants with a choice regarding which risk factor to address resulted in improvement in numerous risk factors simultaneously [34]. Moreover, increasing participant choice has been recommended in text message studies for patients with diabetes [35, 36] and adults in a physical activity study [37]. Incorporating optional features within a program may therefore be a simple solution to increase engagement, acceptability, and feelings of control over one's health.

Implementation of evidence-based interventions can be challenging, even for low-cost, accessible interventions. Participants in the current study suggested that implementation should be a combined effort from credible sources like the government's medical care program, not-for-profit breast cancer organisations, and telecommunications and insurance companies. Moreover, participants expressed that breast cancer treatment and recovery can be financially challenging $[38,39]$, which can lower quality of life [40], making program subsidies important. Participants hypothesised that if the intervention improves health outcomes, it may save the healthcare system money in the long-term. This hypothesis is supported for in-person interventions and some eHealth interventions for people with chronic diseases [41-44]. A recent economic analysis of a 6-month lifestyle-focused text message intervention for secondary prevention of CVD found that reduced hospitalisations due to interventioninduced health improvements could save the government nearly \$10Million [13]. However, further research is needed to elucidate the cost-effectiveness of text message interventions for patients with breast cancer.

The current study has limitations. The mixed-methods evaluation was based on participation in a single-site RCT in Sydney, Australia, which recruited an ethnically diverse sample of breast cancer survivors. The sample size was small, and non-English-speaking participants were excluded; hence, qualitative feedback was limited to people with sufficient English communication skills. Moreover, the factors influencing engagement may differ among the wider population, as people willing to participate in a health-related RCT may be inherently more willing to click on links or desire additional resources. However, suggestions for improvement focused on optional program interactive features (weblinks, text message replies), so that future participants could choose the level of preferred engagement with EMPOWERSMS. Conducting focus groups via videoconference may have limited some people's ability to participate due to technology barriers, such as age or Internet connection. However, the participants' age range was broad (36-67 years) and only one participant reported being unable to attend due to Internet instability (age 52 years). Since COVID-19 has resulted in the cancellation of many in-person oncology appointments, telehealth and videoconference appointments have been becoming more common and acceptable among breast cancer patients [45] due to their convenience and eliminating travel time. Our study demonstrates that videoconference focus groups are possible. However, option to attend via telephone can reduce access barriers for those without a strong Internet connection or technology skills. Focus group participants also received $\$ 20$ for their 1-h participation. However, the Australian national minimum wage in 2019 was $\$ 19.49$ per hour [46], 12/16 (75\%) participants were working full-time, and 6/16 (37.5\%) participants opted to donate their $\$ 20$ to a local breast cancer charity, making it unlikely that the compensation was a main reason for participation. Although there is growing evidence that text message programs are acceptable, useful, and effective at encouraging health-promoting behaviours in patients with a variety of chronic diseases in numerous countries $[9,11$, $37,47-50]$ and can be easily co-designed and delivered in different languages [20,51], further large-scale research studies are needed for patients with breast cancer in other geographical regions and non-English speakers. 


\section{Conclusion}

The mixed-methods process evaluation found that a lifestylefocused text message program was wide-reaching, accessible, acceptable, and useful for women recovering from breast cancer treatment. The program has potential to improve continuity of care between medical appointments. Factors influencing engagement and enablers for future interventions include adding more weblinks to credible health information and credible medical staff or not-for-profit organisations offering the program and providing subsidised to reduce financial barriers.

Acknowledgements A warm thank you to the breast cancer consumer representatives from Breast Cancer Network Australia and Westmead Breast Cancer Institute (WBCI) and all staff and patients at the WBCI for their involvement in the study, especially administration staff for their support (Mrs Kerry Prior, Mrs Seena Jayaraj, Mrs Lalitha Chemudapati, Mrs Zenaida Bables, Mrs Swapna Paravalappil). A special thank you to consumer representatives Christine Mitchell and Fiona Neill and breast care nurses Jenny Cooper and Sarah Laffin. Thank you to staff members of the Westmead Applied Research Centre and Engagement and Co-design Research Hub for their research support, especially Caroline $\mathrm{Wu}$.

Author contribution AS, JR, SRP, and EE contributed to the study conception and design. AS, RR, MH, CCK, AT, KM, KAS, EE, SRP, and JR contributed to the intervention development. AS and RR supported participant recruitment and data collection. AS, RR, SCMS, JTK, and KK contributed to the analyses of the results. AS, JR, SRP, and RR drafted the manuscript. All authors commented on previous versions of the manuscript and have read and approved the final manuscript.

Funding The study was supported by the New South Wales Government Agency for Clinical Innovation Research Grant Scheme, and several team members are supported by scholarships and fellowships. AS's stipend is provided by the University of Sydney's Research Training Program Scholarship and a Supplementary Postgraduate Research Scholarship in Breast Cancer. SRP is supported by a National Health and Medical Research Council Early Career Fellowship (APP1157438) and a National Heart Foundation Postdoctoral Fellowship (HF102164). $\mathrm{KH}$ is supported by a NHMRC Investigator Grant (Emerging leadership 1) (APP1196724). JR is supported by a National Health and Medical Research Council Career Development Fellowship (APP1143538). CKC is supported by a NHMRC Investigator Grant (APP1195326).

Data availability The datasets generated during and/or analysed during the current study are available from the corresponding author on reasonable request.

\section{Declarations}

Ethics approval All procedures performed in studies involving human participants were in accordance with the ethical standards of the institutional and/or national research committee and with the 1964 Helsinki Declaration and its later amendments or comparable ethical standards. The study was approved by the Western Sydney Local Health District Human Research Ethics Committee (HREC; AU RED HREC/18/ WMEAD/281).

Consent to participate All participants provided written informed consent, and ethics approval was obtained from the Western Sydney
Local Health District Human Ethics Research Committee (AU RED HREC/18/WMEAD/281).

Conflict of interest The authors declare no competing interests.

\section{References}

1. Bray F, et al. Global cancer statistics 2018: GLOBOCAN estimates of incidence and mortality worldwide for 36 cancers in 185 countries. CA: Cancer J Clin. 2018;68(6):394-424.

2. Mols F, et al. Quality of life among long-term breast cancer survivors: a systematic review. Eur J Cancer. 2005;41(17):2613-9.

3. Leysen L, et al. Risk factors of pain in breast cancer survivors: a systematic review and meta-analysis. Support Care Cancer. 2017;25(12):3607-43.

4. Keesing S, McNamara B, Rosenwax L. Cancer survivors' experiences of using survivorship care plans: a systematic review of qualitative studies. J Cancer Surviv. 2015;9(2):260-8.

5. Miedema B, Easley J. Barriers to rehabilitative care for young breast cancer survivors: a qualitative understanding. Support Care Cancer. 2012;20(6):1193-201.

6. Helm EE, Kempski KA, Galantino MLA. Effect of disrupted rehabilitation services on distress and quality of life in breast cancer survivors during the COVID-19 pandemic. Rehabilitation Oncology. 2020;38(4):153-8.

7. Rittberg R, et al. Canadian cancer centre response to COVID19 pandemic: a national and provincial response. Curr Oncol. 2021;28(1):233-51.

8. Agyapong VIO, et al. Supportive text messaging for depression and comorbid alcohol use disorder: single-blind randomised trial. J Affect Disord. 2012;141(2):168-76.

9. Chow CK, et al. Effect of lifestyle-focused text messaging on risk factor modification in patients with coronary heart disease: a randomized clinical trial. JAMA. 2015;314(12):1255-63.

10. Hall AK, Cole-Lewis H, Bernhardt JM. Mobile text messaging for health: a systematic review of reviews. Annu Rev Public Health. 2015;36(1):393-415.

11. Agyapong VIO, et al. Changes in stress, anxiety, and depression levels of subscribers to a daily supportive text message program (Text4Hope) during the COVID-19 pandemic: cross-sectional survey study. JMIR Ment Health. 2020;7(12):e22423.

12. Redfern J, et al. Factors influencing engagement, perceived usefulness and behavioral mechanisms associated with a text message support program. PloS One. 2016;11(10):e0163929.

13. Burn E, Nghiem S, Jan S, Redfern J, Rodgers A, Thiagalingam A, ... \& Chow CK. Cost-effectiveness of a text message programme for the prevention of recurrent cardiovascular events. Heart. 2017;103(12):893-894.

14. Singleton A, R.R., Tat-Ko J, Che Mun Sum,S, Hafiz, Partridge SR, Hyun KK, Sherman K, Elder E, and Redfern J., eHealth strategies to improve health outcomes among patients with breast cancer: a systematic review and meta-analysis. International Psychosocial Oncology Society's 22nd World Congress of Psycho-Oncology and Psychosocial Academy, 2021.

15. Brett $\mathbf{J}$, et al. Mapping the impact of patient and public involvement on health and social care research: a systematic review. Health Expect. 2014;17(5):637-50.

16. Tsianakas $\mathrm{V}$, et al. Implementing patient-centred cancer care: using experience-based co-design to improve patient experience in breast and lung cancer services. Support Care Cancer. 2012;20(11):2639-47. 
17. Mead KH, et al. Identifying patients' priorities for quality survivorship: conceptualizing a patient-centered approach to survivorship care. J Cancer Surviv. 2020;14(6):939-58.

18. Nekhlyudov L, et al. Developing a quality of cancer survivorship care framework: implications for clinical care, research, and policy. JNCI: J Nat Cancer Ins. 2019;111(11):1120-30.

19. NHaMR C. Statement on consumer and community involvement in health and medical research. In Consumers Health Forum of Australia 2016.

20. Singleton A, et al. Co-designing a lifestyle-focused text message intervention for women after breast cancer treatment: mixed methods study. J Med Int Res. 2021;23(6):e27076.

21. Singleton A, et al. A text message intervention to support women's physical and mental health after breast cancer treatments (EMPOWER-SMS): a randomised controlled trial protocol. BMC Cancer. 2019;19(1):660.

22. Barbour R. Doing focus groups 2nd edition. (Vol. 4) Sage Publications Ltd. 2008

23. Weinkove $\mathrm{R}$, et al. Managing haematology and oncology patients during the COVID-19 pandemic: interim consensus guidance. Med J Aust. 2020;212(10):481-9.

24. Smith J, Firth J. Qualitative data analysis: the framework approach. Nurse Res. 2011;18(2):52-62.

25. Vroman KG, Arthanat S, Lysack C. "Who over 65 is online?" Older adults' dispositions toward information communication technology. Comput Hum Behav. 2015;43:156-66.

26. Cox A, et al. Cancer survivors' experience with telehealth: a systematic review and thematic synthesis. J Med Internet Res. 2017;19(1):e11.

27. Junod Perron N, et al. Text-messaging versus telephone reminders to reduce missed appointments in an academic primary care clinic: a randomized controlled trial. BMC Health Serv Res. 2013;13(1):125.

28. Head KJ, et al. Efficacy of text messaging-based interventions for health promotion: a meta-analysis. Soc Sci Med. 2013;97:41-8.

29. Armanasco AA, et al. Preventive health behavior change text message interventions: a meta-analysis. Am J Prev Med. 2017;52(3):391-402.

30. Izuma K, et al. Neural correlates of cognitive dissonance and choice-induced preference change. Proc Natl Acad Sci. 2010;107(51):22014-9.

31. Paterson RJ, Neufeld RWJ. What are my options?: influences of choice availability on stress and the perception of control. J Res Pers. 1995;29(2):145-67.

32. Whelan T, et al. Effect of a decision aid on knowledge and treatment decision making for breast cancer surgery a randomized trial. JAMA. 2004;292(4):435-41.

33. BorjAlilu S, et al. Exploring the role of self-efficacy for coping with breast cancer: a systematic review. Arch Breast Cancer [Internet]. 2017;4(2):42-57. https://doi.org/10.19187/abc.20174 $242-57$

34. Redfern J, et al. Choice of secondary prevention improves risk factors after acute coronary syndrome: 1-year follow-up of the CHOICE (choice of health options in prevention of cardiovascular events) randomised controlled trial. Heart. 2009;95(6):468-75.
35. Dobson R, et al. Diabetes text-message self-management support program (SMS4BG): a pilot study. JMIR mHealth uHealth. 2015;3(1):e32.

36. Mulvaney SA, Ritterband LM, Bosslet L. Mobile intervention design in diabetes: review and recommendations. Curr Diab Rep. 2011;11(6):486-93.

37. Smith DM, et al. Text message interventions for physical activity: a systematic review and meta-analysis. Am J Prev Med. 2020;58(1):142-51.

38. Sun L, et al. Global treatment costs of breast cancer by stage: a systematic review. PloS one. 2018;13(11):e0207993.

39. Gordon L, et al. Exploring the economic impact of breast cancers during the 18 months following diagnosis. Psychooncology. 2007;16(12):1130-9.

40. Meneses K, et al. Does economic burden influence quality of life in breast cancer survivors? Gynecol Oncol. 2012;124(3):437-43.

41. Shields GE, et al. Cost-effectiveness of cardiac rehabilitation: a systematic review. Heart. 2018;104(17):1403-10.

42. LLiu S, Zhao Q, Li W, Zhao X, \& Li K. The cost-effectiveness of pulmonary rehabilitation for COPD in different settings: a systematic review. Appl Health Econ Health Policy. 2021;19(3):313-324.

43. Kooij L, Groen WG, van Harten WH. The effectiveness of information technology-supported shared care for patients with chronic disease: a systematic review. J Med Internet Res. 2017;19(6):e221.

44. Parkinson B, et al. Cost-effectiveness of the BRECONDA decision aid for women with breast cancer: results from a randomized controlled trial. Psychooncology. 2018;27(6):1589-96.

45. Burbury $\mathrm{K}$, et al. Telehealth in cancer care: during and beyond the COVID-19 pandemic. Intern Med J. 2021;51(1):125-33.

46. Commission, A.F.W., National minimum wage order 2019. Fair Work Act 2009 - Annual Wage Review 2018-19, 2019. Accessed on 05/08/2021: https://www.fwc.gov.au/documents/wage-reviews/ 2018-19/decisions/c20191-order.pdf

47. Hurt $\mathrm{K}$, et al. mHealth interventions in low and middleincome countries: a systematic review. Glob J Health Sci. 2016;8(9):54429.

48. Mougalian SS, et al. Bidirectional text messaging to monitor endocrine therapy adherence and patient-reported outcomes in breast cancer. JCO Clinical Cancer Informatics. 2017;1:1-10.

49. Rico TM, et al. Text messaging (SMS) helping cancer care in patients undergoing chemotherapy treatment: a pilot study. J Med Syst. 2017;41(11):181.

50. Spoelstra SL, et al. Feasibility of a text messaging intervention to promote self-management for patients prescribed oral anticancer agents. Oncol Nurs Forum. 2015;42(6):647-57.

51. Godino JG, et al. Text messaging and brief phone calls for weight loss in overweight and obese English-and Spanish-speaking adults: a 1-year, parallel-group, randomized controlled trial. PLoS Med. 2019;16(9):e1002917.

Publisher's Note Springer Nature remains neutral with regard to jurisdictional claims in published maps and institutional affiliations. 